\title{
Diabetic neuropathy: a risk factor for severe COVID-19?
}

\author{
Dario Pitocco $^{1,3} \cdot$ Luca Viti $^{1}$ (D) Angelo Santoliquido ${ }^{2} \cdot$ Linda Tartaglione $^{1} \cdot$ Mauro Di Leo $^{1} \cdot$ Antonio Bianchi $^{3}$. \\ Salvatore Caputo ${ }^{1}$. Alfredo Pontecorvi ${ }^{3}$
}

Received: 11 November 2020 / Accepted: 9 December 2020 / Published online: 3 February 2021

(c) Springer-Verlag Italia S.r.l., part of Springer Nature 2021

Respiratory viruses are associated with more severe symptoms and complications in diabetic subjects. We hypothesize that the diabetic neuropathy could provide a significant contribution to risk of disease severity. The diabetic neuropathy and the autonomic dysfunction might determine the loss of immune response regulation and contribute to reduction of pulmonary function, in particular gas exchange.

Preliminary data highlight how the immune system reaction causes a lung damage during COVID-19 infection. The exaggerated cytokine production, which currently represents the most interesting therapeutic target, is a pathophysiological mechanism shared with other severe acute respiratory infections, such as SARS and MERS [1]. T-lymphopenia is usually detectable in COVID-19 patients, caused by consumption of CD4 + and CD8 + lymphocytes and decrease in regulatory $\mathrm{T}$ cells, with a loss of suppression of inappropriate immune response. Moreover, a higher serum concentration of pro-inflammatory cytokines (TNF-alpha, IL-6) and chemokines (Il-8) is frequently found in severe forms of COVID-19 [2]. These data suggest a role for the inflammatory response in the development of severe complications of the disease.

Nervous system regulates innate and adaptive immunity through a mechanism called the inflammatory reflex. The autonomic nervous system receives sensory inputs from

Managed by Antonio Secchi.

Dario Pitocco and Luca Viti have contributed equally to the manuscript.

Luca Viti

Lucaviti7@gmail.com

1 Diabetes Care Unit, Fondazione Policlinico A.Gemelli IRCCS, Roma, Italy

2 Department of Vascular Medicine, Fondazione Policlinico A.Gemelli IRCCS, Roma, Italy

3 Department of Endocrinology, Fondazione Policlinico A. Gemelli IRCCS, Roma, Italy inflammatory cells and modulates the immune response by a negative feedback mechanism. Humoral or neural signals can activate the sensory afferent arc of the reflex. TNF-alpha and the other inflammatory mediators can gain access to central nervous system through circumventricular organs, which are characterized by discontinuities of blood-brain barrier. Furthermore, sensory innervation of immune organs by ascending vagus fibres can send afferent signals to the reflex. The motor efferent arc of the reflex releases acetylcholine in the reticuloendothelial system, leading to decrease in production of pro-inflammatory cytokines in macrophages [2]. Diabetic neuropathy could play a more important role in the loss of capacity to regulate immune response, since some physiological mechanisms could be impaired with a consequent overexpressed response to inflammatory stimuli. Our hypothesis is that the dysfunction of the autonomic nervous system, reflecting an imbalance between sympathetic and parasympathetic modulation, could affect the proinflammatory state in diabetic patients with COVID-19, as a consequence of a dysregulation of the inflammatory reflex.

Regarding lung function, we showed a significant association between autonomic nervous system dysfunction and reduced DLCO in type 1 diabetes [1]. One of the most frequently described histopathological pulmonary alterations in diabetes is the thickening of the pulmonary capillary basal laminae and of the alveolar epithelium, which correlates with the severity of microvascular complications [1]. An increase in collagen protein synthesis and accelerated cross-linking, induced by reactive oxygen species-induced oxidative stress, together with non-enzymatic protein glycation in the extracellular matrix, seems to cause injury in the pulmonary microcirculation by increasing vessel wall thickening. However, we found that DLCO significantly correlated with HRV (heart rate variability), even after adjustment for clinical and metabolic variables, such as microvascular complications [3]. A possible explanation is that diabetic autonomic neuropathy could induce functional alterations in the regulation of pulmonary bronchomotor tone as well as in the control of ventilation [4]. In another study, we reported 
changes in bronchomotor tone among diabetic patients, who showed a faster clearance of I123 MIBG during ventilatory scintigraphy, as a result of noradrenergic dysfunction in bronchial innervation [5].

Therefore, in this setting it might be useful to investigate the correlation between severity of COVID-19 pneumonia and diabetic autonomic neuropathy to confirm our hypothesis and support the finding of a different management.

Funding The authors did not receive any specific grant from funding agencies in the public, commercial, or not-for-profit sectors.

\section{Compliance with ethical standards}

Conflict of interest The authors declare no conflict of interest.

\section{References}

1. Tracey KJ (2020) The inflammatory reflex. Nat 420(6917):853-859
2. Qin $\mathrm{C}$, Zhou L, Hu Z, Zhang S, Yang S, Tao Y, Xie C, Ma K, Shang K, Wang W, Tian DS (2020) Dysregulation of immune response in patients with COVID-19 in Wuhan China. Clin Infect Dis. https://doi.org/10.2139/ssrn.3541136

3. Pitocco D, Santangeli P, Fuso L, Zaccardi F, Longobardi A, Infusino F, Incalzi RA, Lanza GA, Crea F, Ghirlanda G (2008 Nov) Association between reduced pulmonary diffusing capacity and cardiac autonomic dysfunction in type 1 diabetes. Diabet Med 25(11):1366-1369

4. Pitocco D, Fuso L, Conte EG, Zaccardi F, Condoluci C, Scavone G, Incalzi RA, Ghirlanda G (2012) The diabetic lung-a new target organ? Rev Diabet Stud 9(1):23-35

5. Antonelli Incalzi R, Fuso L, Giordano A, Pitocco D, Maiolo C, Calcagni ML, Ghirlanda G (2002) Neuroadrenergic denervation of the lung in type I diabetes mellitus complicated by autonomic neuropathy. Chest 121(2):443-451

Publisher's Note Springer Nature remains neutral with regard to jurisdictional claims in published maps and institutional affiliations. 